\title{
Low-Energy QCD: Few-Nucleon Research at TUNL
}

\author{
Calvin R. Howell* \\ Department of Physics, Duke University and the Triangle Universities Nuclear Laboratory, \\ Durham, NC, USA \\ E-mail: howellatunl.duke.edu
}

The main goal of few-nucleon research at the Triangle Universities Nuclear Laboratory (TUNL) is to perform high-precision measurements that contribute to advancing ab-initio calculations and testing nucleon-interaction models. The current focus is on the three-nucleon system, which has highly developed theory and has sufficient complexity to exhibit influences of threenucleon forces.The few-nucleon measurements performed at the High Intensity Gamma-ray Source $(\mathrm{HI} \gamma \mathrm{S})$ and in the tandem lab provide complementary information. We have performed the first exclusive differential cross-section measurements for photodisintegration of ${ }^{3} \mathrm{He}$ at low energy. The measurements were carried out at an incident beam energy of $15 \mathrm{MeV}$. A difference in the ${ }^{1} \mathrm{~S}_{0}$ neutron-proton scattering length determined in this reaction from the value established by two-nucleon scattering data would be potential evidence for three-nucleon interactions that are not included in current calculations. The strength of the neutron-neutron $(n n){ }^{1} \mathrm{~S}_{0}$ interaction is directly evaluated using $n n$ quasi-free scattering in neutron-deuteron breakup. We are performing measurements of this process at two incident neutron beam energies, 10 and $16 \mathrm{MeV}$, using substantially different detector setups. The experiment methods and preliminary results of these experiments will be presented.

The 9th International workshop on Chiral Dynamics

17-21 September 2018

Durham, NC, USA

${ }^{*}$ Speaker. 


\section{Introduction}

The properties of nuclei and nuclear reactions inform us about details of the strong nuclear force, which is a residue resulting from the interaction of quarks and gluons at confinement-scale distances where color forces are strong, i.e., in the non-perturbative regime of QCD at distances of order the size of nucleons. Effective field theories (EFT), e.g., chiral perturbation theory, and Lattice QCD calculations provide theoretical frames for connecting QCD to low-energy nuclear phenomena. Organizing goals in low-energy QCD research include improving the precision of determinations of the macroscopic structure properties of nucleons, expanding descriptions of the bound-state properties of light nuclei, and advancing the accuracy of predictions of the cross sections for few-nucleon reactions. In particular, high-precision studies of bound-state properties of light nuclei and reaction dynamics in few-nucleon systems allow for assessments of the theoretical treatment of nuclear interactions over a wide range of phase space. Interpretation of few-nucleon reactions data with ab-initio calculations provide feedback to theorists that is essential for refining rigorous treatments of strong interactions in the nuclear medium.

$\mathrm{Ab}$-initio calculations of the binding energy of the ground state and the excitation spectrum of light nuclei [1, 2, 3, 4] and of the cross section for few-nucleon reactions [5] enable refinement of two- and three-nucleon interactions using effective degrees of freedom. Current models of the strong nuclear force (two- and three-nucleon interactions) include semi-empirical potential models, e.g., Refs. $[6,7,8,9,10]$, effective field theory formulations of two-nucleon ( $2 \mathrm{~N})$ and three-nucleon $(3 \mathrm{~N})$ interactions [11, 12], and Lattice QCD (LQCD) calculations of few-nucleon systems, e.g., see Refs. [13, 14]. Discrepancies between data and theory predictions provide circumstantial evidence of effects due to three-nucleon interactions (3NIs). Examples include the resolution of the tritonbinding energy discrepancy $[9,7]$ and systematic studies of the ground and excited states of light nuclei using Monte-Carlo Green function simulations [1] and no-core shell model calculations $[2,3,4]$. Also, in the calculation of the nuclear equation of state, the energy per nucleon as a function of density is highly sensitive to the inclusion of 3NIs, especially at high nuclear densities relevant to the interior of neutron stars [15].

Because 3NIs are most important at short distances, the sensitivity of low-energy scattering data to 3 NIs is more subtle than for bound-state properties or quantities measured at high nuclear densities. For this reason, high precision data and theory for a wide variety of scattering quantities are required to make progress on understanding the role of 3NIs in low-energy nuclear reactions. The focus of this session is on the applications of few-nucleon systems to probe detailed features of two- and three-nucleon interactions. The general approach is to use ab-initio calculations of few-nucleon systems to search for quantities that are sensitive to the details of 3NIs and to specific aspects the two-nucleon interaction (2NI).

Four of the five papers in this session described the use of few-nucleon reaction data to probe the effects of 3NIs in scattering processes. Though the initial motivation for the neutron-deuteron breakup, $\mathrm{D}(\mathrm{n}, 2 \mathrm{n})$, cross-section measurements presented by Voinov was for applications in nuclear fusion energy research, the experiment provides a unique and high-accuracy data set that can be used in broad surveys for investigating 3NIs in the few-nucleon continuum. Sekiguchi describes the measurement and interpretation of cross-section and polarization data for deuteron-proton $(d p)$ and proton- ${ }^{3} \mathrm{He}$ elastic scattering at medium energies. The comparison of both cross-section and 
polarization data to ab-inito calculations show clear evidence for $3 \mathrm{NI}$ effects. Their results indicate a spin dependence in the 3NIs. Examples of their results for $d p$ elastic scattering are in Ref. [16]. Skibinski et al. presented comparisons of ab-initio three-nucleon calculations to nucleon-deuteron elastic scattering data at 65 and $200 \mathrm{MeV}$ incident laboratory nucleon energy. The calculations were performed using two chiral 2NIs: the N4LO+ SMS (semilocal momentum-space) model from the Bochum group [17] and the N4LO Moscow(Idaho)-Salamanca potential [18]. These calculations did not include 3 NIs. The quality of the description of the data by theory is suggestive of the need for 3NIs. Viviani presented two sensitivity studies of 3NIs in few-nucleon reactions: (1) the 3N contact terms appearing in chiral perturbation theory at N4LO, and (2) the sensitivity on the values of the N2LO coupling constants $C_{D}$ and $C_{E}$ appearing at N2LO in the $3 \mathrm{NI}$. These studied were carried out using three-nucleon and four-nucleon scattering data for cross sections and polarization quantities.

The main focus of the few-nucleon research program at the Triangle Universities Nuclear Laboratory (TUNL) is on using three-nucleon ( $3 \mathrm{~N}$ ) reactions to probe for effects due to 3NIs. This paper describes two experiments that are underway at the TUNL. One is carried out using the nearly mono-energetic neutron beam in the tandem laboratory, and the other is performed using the Compton photon beam at the High Intensity Gamma-ray Source $(\mathrm{HI} \gamma \mathrm{S})$. These experiments provide complementary information in evaluating ab-initio calculations. S-wave neutron-neutron $(n n)$ quasi-free scattering (QFS) in neutron-deuteron $(n d)$ breakup is measured at two neutron beam energies, 10 and $16 \mathrm{MeV}$. Discrepancies between data and theory suggest that 3 NIs might be important. The first exclusive differential cross sections for photodisintegration of ${ }^{3} \mathrm{He}$ at low energy have been measured at $\mathrm{HI} \gamma \mathrm{S}$ at an incident beam energy of $15 \mathrm{MeV}$. The focus of the analysis is on determining the cross sections for the ${ }^{1} \mathrm{~S}_{0}$ neutron-proton ( $n p$ ) final-state interaction (FSI) and for the collinear configuration in which the neutron is at rest in the lab and the two protons are emitted along the same line in opposite directions. This paper will present the status of the experiments at TUNL on $n n$ QFS in $n d$ breakup and photodisintegration of ${ }^{3} \mathrm{He}$.

\section{Cross-section Measurements of $n n$ QFS in $n d$ Breakup at TUNL}

An analysis by Witała and Glöckle [19] showed that the discrepancy between calculations and the cross-section data for $n n$ QFS in $n d$ breakup can be resolved by increasing the strength of the CDBonn $n n$ s-wave potential [6] by about $8 \%$. This adjustment of the nn interaction strength introduces an unrealistically large charge-symmetry breaking in the $2 \mathrm{~N}$ interaction. Other possible explanations are that 3 NIs that are not included in current calculations are important or that the systematic uncertainties of the published data are underestimated.

We are performing new $n d$ breakup measurements for the purpose of confirming the reported cross sections. The measurements are performed in the tandem accelerator laboratory at TUNL. The experiment setup for our measurements at $10 \mathrm{MeV}$ is shown in Fig. 1. The neutron beam was produced via the ${ }^{2} \mathrm{H}(\mathrm{d}, \mathrm{n})^{3} \mathrm{He}$ reaction with a pulsed deuteron beam (period $=400 \mathrm{~ns}, \mathrm{FWHM}=2$ ns) incident on a 3.16-cm-long cylindrical cell filled with deuterium gas to a pressure of $5 \mathrm{~atm}$. The resulting neutron beam had a centroid energy of $10.0 \mathrm{MeV}$ with an energy spread of 330 $\mathrm{keV}$ (full width). Scattered neutrons were detected by two heavily shielded liquid scintillators positioned on opposite sides of the beam axis at equal angles. The two detectors are $5.08 \mathrm{~cm}$ 


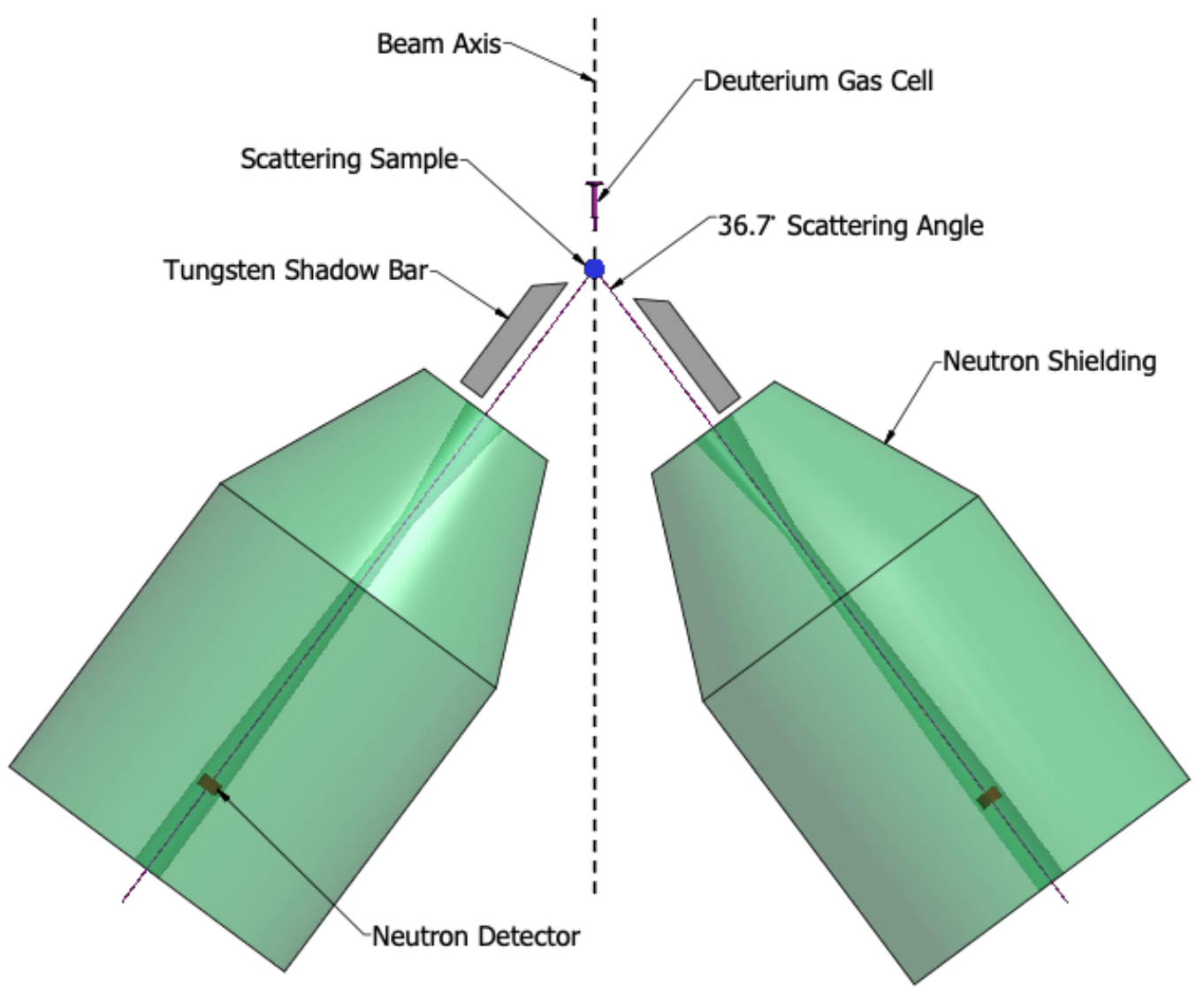

Figure 1: A diagram of the experiment setup (distances are to scale) for the $n n$ QFS measurements at 10 $\mathrm{MeV}$. The sample is $12.4 \mathrm{~cm}$ from the center of the neutron production cell, and the detectors are placed about $265 \mathrm{~cm}$ from the target at $36.7^{\circ}$ on either side of the beam.

long cylinders with diameters of $12.7 \mathrm{~cm}$ (beam left) and $8.89 \mathrm{~cm}$ (beam right). The left and right detectors are filled with NE-213 and NE-218 liquid scintillator, respectively. Each detector is housed inside a cylindrical shielding enclosure of lithium-doped paraffin with a double-truncated conical copper collimator [20]. Tungsten shadow bars were positioned to shield the detectors from directly viewing the neutron production cell. The distance from the center of the sample to the center of each detector was $264.9 \mathrm{~cm}$ for the left detector and $264.3 \mathrm{~cm}$ for the right detector. The target was a solid deuterated polyethylene $\left(\mathrm{CD}_{2}\right)$ cylinder with dimensions $28 \mathrm{~mm}$ (diameter) $\times 36$ $\mathrm{mm}$ (height). The mass of the sample was $25.2 \mathrm{~g}$. In this geometry, the center-to-center distance between the neutron production cell and the $\mathrm{CD}_{2}$ sample is only about $12 \mathrm{~cm}$. This distance is constrained by the construction of the beam line and the pivot of the detector carriages. The short source-to-sample distance has benefits and disadvantages. The main benefit is the high luminosity obtained by having the target close to the neutron source. The disadvantage is the substantial finitegeometry effects due to the tight geometry. The target-beam integrated luminosity was determined using $n d$ elastic scattering.

The measurements and data analysis for the $10-\mathrm{MeV}$ experiment are complete. A plot of our preliminary data is shown in Fig. 2 in comparison to Monte-Carlo simulations based on $a b$ - 


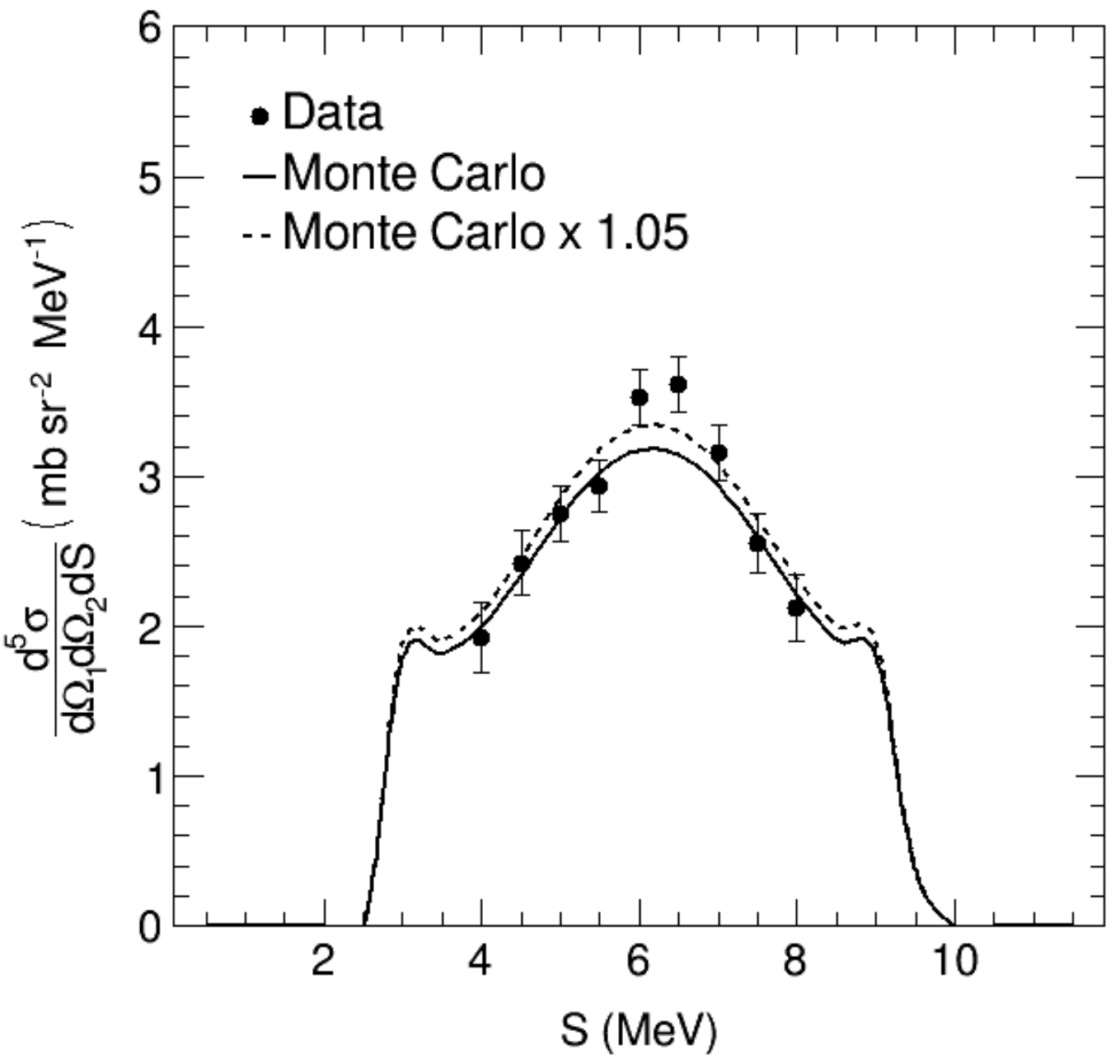

Figure 2: A plot of the preliminary TUNL cross-section data for $n n$ QFS in $n d$ breakup as a function of the kinematic $\mathrm{S}$ curve for incident neutron beam energy of $10 \mathrm{MeV}$ and symmetric $n n$ coincidence angles of $36.7^{\circ}$. The error bars represent statistical uncertainties only. The solid curve is a Monte-Carlo simulation based on ab-initio nd breakup calculations of Witała that use the CD-Bonn nucleon-nucleon potential model [6]. The dashed curve is the solid curve multiplied by a factor of 1.05 to obtain the minimum chi-square fit to the data with the simulated cross section.

initio nd breakup calculations. The systematic error in the data is $\pm 6 \%$, which is due mostly to the uncertainties in the neutron detection efficiency and the target-beam luminosity. Considering this systematic uncertainty, our data are in agreement with the ab-initio $3 \mathrm{~N}$ calculations, thus, validating our experimental techniques. These results are contrary to previous measurements that were performed around $26 \mathrm{MeV}$ [21, 22].

The next set of measurements will be carried out at $16 \mathrm{MeV}$ using a different experimental setup, which is shown in Fig. 3. In this setup, the collimated neutron beam allows the use of unshielded detectors. With this arrangement, the $n n$ QFS process will be measured at several scattering angles simultaneously. The neutrons are produced using the ${ }^{2} \mathrm{H}(\mathrm{d}, \mathrm{n})$ reaction as with the $10-\mathrm{MeV}$ measurements. However, in this experiment configuration, the $\mathrm{CD}_{2}$ sample is approxi- 


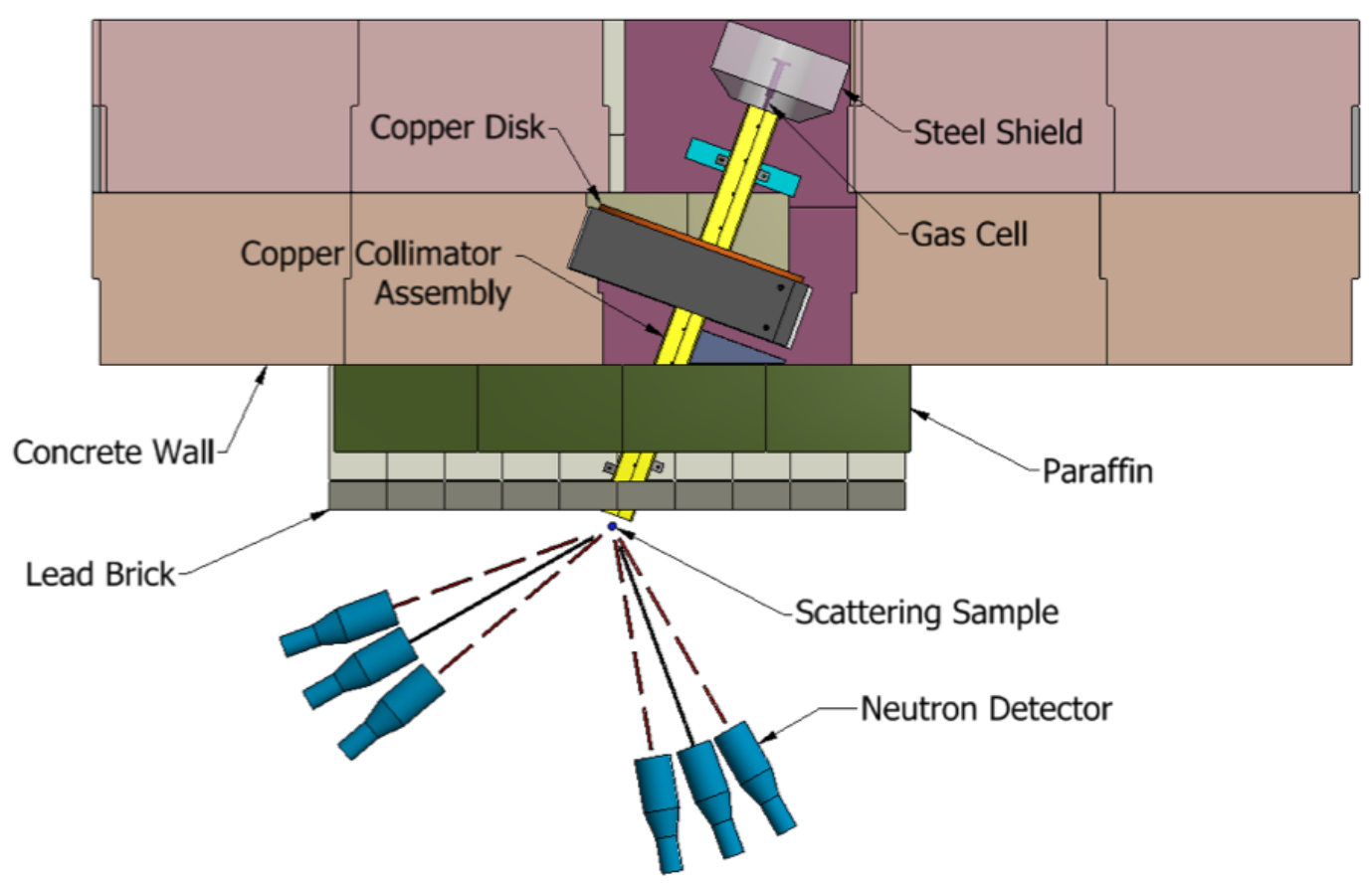

Figure 3: A diagram of the experiment setup for the 16-MeV measurements. The neutron beam is collimated and the detectors are unshielded. The sample is about $160 \mathrm{~cm}$ from the neutron production source, and the distance from the $\mathrm{CD}_{2}$ sample to the neutron detectors is about $75 \mathrm{~cm}$.

mately $160 \mathrm{~cm}$ from the center of the neutron production source. The center-to-center distance between the sample and the neutron detectors is about $75 \mathrm{~cm}$. This setup is similar to that used in previous experiments [21,22], thereby allowing a direct comparison of the results of our experiment with previous measurements. The sources of the systematic uncertainties in the two setups are substantially different. Measurements with these two very different experiment setups are intended to evaluate the accuracy our experiment simulations that are used to account for finite geometry effects.

\section{Exclusive Photodisintegration of ${ }^{3} \mathrm{He}$ at TUNL}

Photonuclear reactions on few-nucleon systems are sensitive to meson exchange currents (MEC) that contribute to pairwise nucleon-nucleon interactions and to $3 \mathrm{~N}$ current operators, which are associated with 3NIs. We are performing simultaneous cross-section measurements of twoand three-body photodisintegration of ${ }^{3} \mathrm{He}$ using the high-intensity and nearly mono-energetic $\gamma$ ray beam at $\mathrm{HI} \gamma \mathrm{S}$. This experiment will produce the first cross-section data of kinematically complete, i.e., exclusive, three-body photodisintegration of ${ }^{3} \mathrm{He}$ at low energies. A 3D rendering of the experiment setup is shown in Fig. 4. An example of the signal-to-background quality of the charged-particle coincidence spectra is illustrated in Fig. 5. The data have been collected and analysis is underway to compare experiment to ab-initio $3 \mathrm{~N}$ calculations by Witala [23] and Deltuva [24]. 


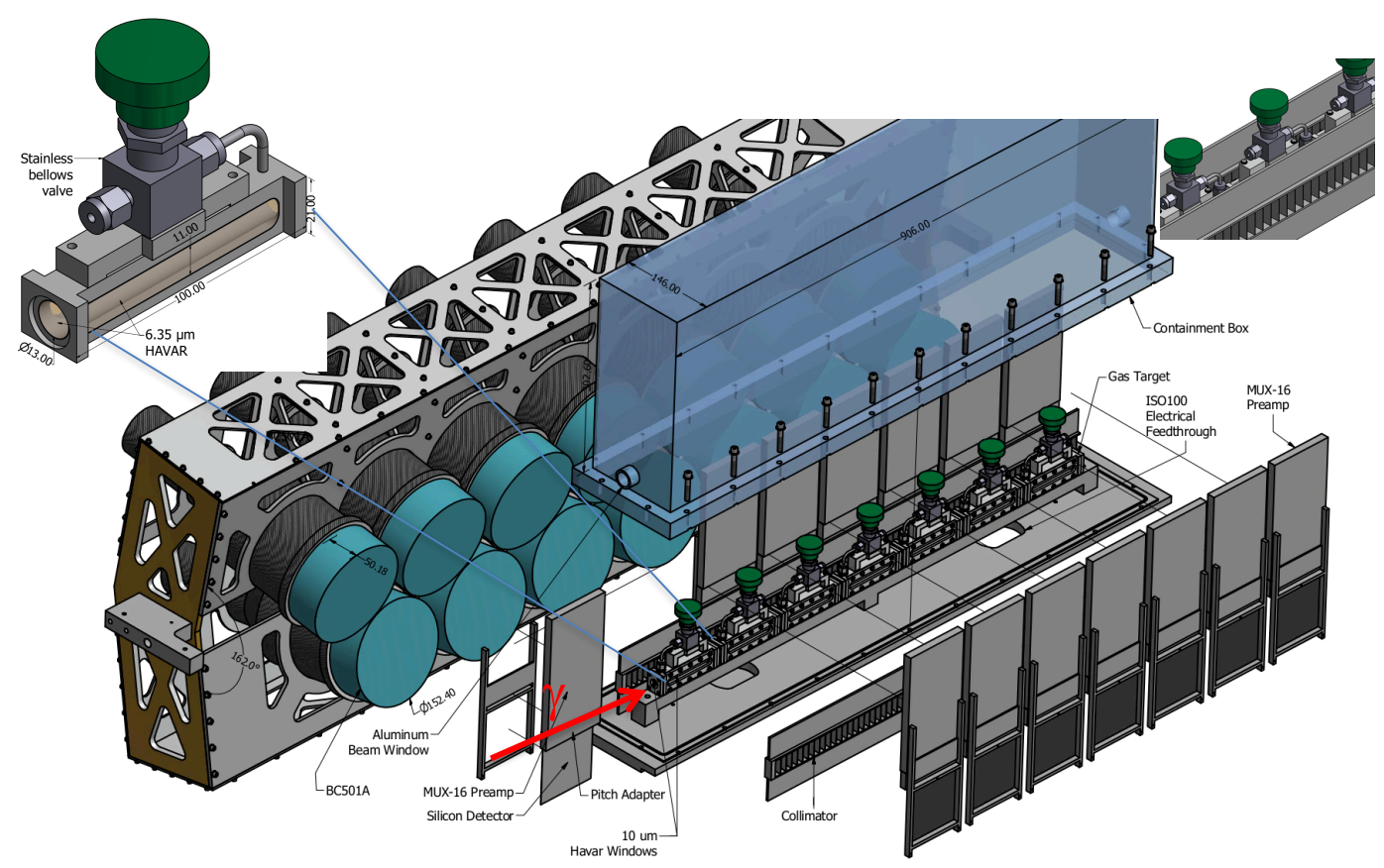

Figure 4: Three-dimensional rendering of the experimental setup used in the ${ }^{3} \mathrm{He}$ photodisintegration measurements at $\mathrm{HI} \gamma \mathrm{S}$. The setup consists of an array of $14 \mathrm{BC}-501$ liquid scintillators $(12.5 \mathrm{~cm} \mathrm{dia.} \times 5 \mathrm{~cm}$ thick) on each side of the beam axis for neutron detection, 7 collimated silcon-strip detectors on each side of the beam axis for proton and deuteron detection, and $7{ }^{3} \mathrm{He}$ gas targets with $10-\mu \mathrm{m}$ thick Havar windows. The targets are $10 \mathrm{~cm}$ long and pressurized with $5 \mathrm{~atm}$ of ${ }^{3} \mathrm{He}$ gas. The area of each silicon strip is $5.9 \mathrm{~mm} \times$ $91.6 \mathrm{~mm}$, and each panel has 16 strips. The neutron detectors are shown only on one side of the beam axis. The average distance between the $\gamma$-ray beam axis and the center of a neutron detector is $42 \mathrm{~cm}$.

\section{Summary}

We have measured the cross section for nn QFS in $n d$ breakup at an incident neutron beam energy of $10.0 \mathrm{MeV}$. The main features of the experiment method was the use of an open neutron source, heavily shielded neutron detectors, and time-of-flight techniques to determine the energy of the detected neutrons. Our preliminary data at $10 \mathrm{MeV}$ agree with predictions of ab-initio theoretical calculations within the about $\pm 6 \%$ systematic uncertainty in the data. The good agreement between data and theory indicates that the technique of using $n d$ elastic scattering to determine the beam-target luminosity works well for this type of measurement. Because of the substantial finitegeometry effects of this setup, we are unable to rule out the validity of the reported discrepancies between data and theory for this process [21, 22].

Data have been collected for $n n$ QFS at several scattering angles in $n d$ breakup at $16 \mathrm{MeV}$ incident neutron beam energy. These data were measured using a heavily shielded neutron source and a collimated neutron beam. This experiment setup allowed the use of unshielded detection, thereby enabling simultaneous measurements at several scattering angles.

Data have been collected for the ${ }^{3} \mathrm{He}(\gamma, \mathrm{pp})$ and ${ }^{3} \mathrm{He}(\gamma, \mathrm{pn})$ photodisintegration reactions at $\mathrm{HI} \gamma \mathrm{S}$. This experiment will produce the first cross-section data for exclusive three-body photodisintegration of ${ }^{3} \mathrm{He}$ at low energies where ab-initio few-nucleon calculations are available. The data 


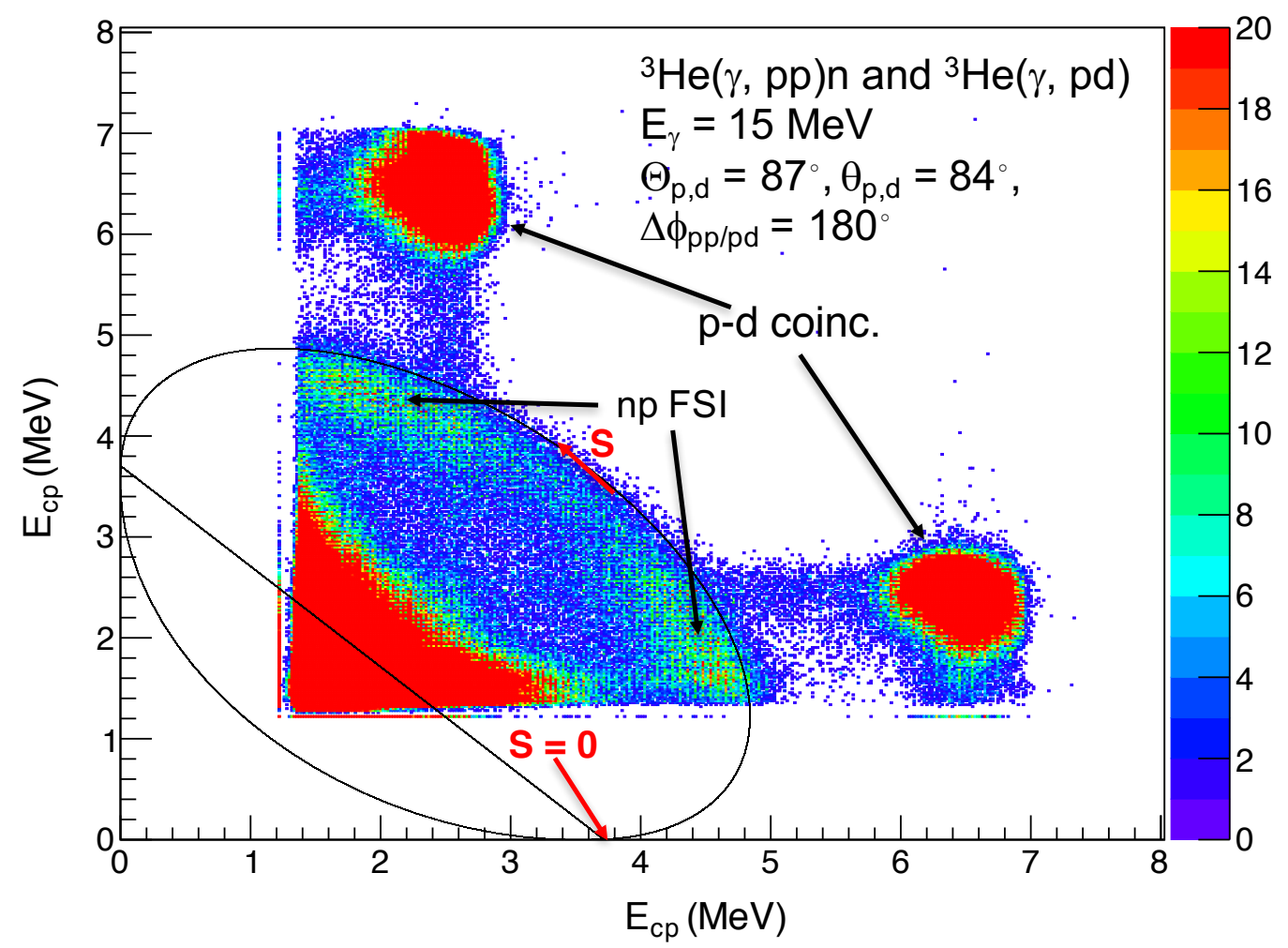

Figure 5: Two-dimensional histogram of the energies for the two charged particles detected in coincidence in photodisintegration of ${ }^{3} \mathrm{He}$ in $\mathrm{HI} \gamma \mathrm{S}$ experiment. The particle scattering angles are given in the plot. The peaks for 2-body photodisintegration and the locus for 3-body photodisintegration are indicated. Also, the cross-section enhancements for the $n p$ final-state interaction (FSI) are identified.

analysis is underway. In the near term, we are considering a followup experiment of this work at a higher incident $\gamma$-ray beam energy. Measuring the full region of the $n p$ FSI cross-section enhancement was limited by the energy threshold of the silicon strip detectors. Increasing the beam energy from 15 to about $30 \mathrm{MeV}$ will give the emitted protons sufficient energy to be detected cleanly above the detector electronic noise level. In the long term (about five year horizon), we are developing an experiment to measure exclusive cross sections for three-body photodisintegration of tritium.

\section{Acknowledgments}

This work is supported in part by the U.S. Department of Energy under grant No. DE-FG0297ER41033 and DE-SC0005367 and by the Polish National Science Center under Grant No. DEC2016/22/M/ST2/00173. The contributions of the following people are appreciated: L.C. Cumberbatch, A.S. Crowell, B.A. Fallin, F.Q.L. Friesen, C.R. Malone, R.C. Malone, D.R. Ticehurst and W. Tornow, Duke University; M.W. Ahmed, D.M. Markoff and B. Crowe, NC Central University; H. Witała, Jagiellonian University; and A. Deltuva, Vilnius University. 


\section{References}

[1] S.C. Pieper and V.R. Pandharipande, Annu. Rev. Nucl. Part. Sci. 51, 53 (2001)

[2] P. Navratil et al., Phys. Rev. Lett. 99, 042501 (2007)

[3] P. Navratil et al., Few-Body Syst. 43, 129 (2008)

[4] A. Nogga, P. Navratil, B.R. Barrett and J.P. Vary, Phys. Rev. C 73, 064002 (2006)

[5] W. Glöckle et al., Phys. Rep. 274, 107 (1996)

[6] R. Machleidt, Phys. Rev. C 63, 024001 (2001)

[7] R.B. Wiringa, V.G.J. Stoks and R. Schiavilla, Phys. Rev. C 51, 38 (1995)

[8] V.G.J. Stoks, R.A.M. Klomp, C.P.F. Terheggen and J.J. de Swart, Phys. Rev. C 49, 2950 (1994)

[9] S.A. Coon, M.D. Scadron and B.R. Barrett, Nucl. Phys. A 242, 467 (1975)

[10] S.C. Pieper, V.R. Pandharipande, R.B. Wiringa and J. Carlson, Phys. Rev. C 64, 014001 (2001)

[11] R. Machleidt and D.R. Entem, Phys. Rep. 503, 1 (2011)

[12] E. Epelbaum, H.-W. Hammer and Ulf-G. Meissner, Rev. Mod. Phys. 81, 1773 (2009)

[13] E. Epelbaum, H Krebs, D. Lee and Ulf-G. Meissner, Phys. Rev. Lett. 106, 192501 (2011)

[14] S.R. Beane et al., Phys. Rev. Lett. 115, 132001 (2015)

[15] P. Danielewicz, AIP Conf. Proc. 597, 24 (2001)

[16] K. Sekiguchi et al., Phys. Rev. C 96, 064001 (2017)

[17] P. Reinert, H. Krebs, E. Epelbaum, Eur. Phys. J. A54, 86 (2018)

[18] D.R. Entem, R. Machleidt, Y. Nosyk, Phys. Rev. C 96, 024004 (2017)

[19] H. Witała and W. Glöckle, Phys. Rev. C 83, 034004 (2011)

[20] D.W. Glasgow et al., Nucl. Instr. Methods 114, 521 (1974)

[21] A. Siepe et al., Phys. Rev. C 65, 034010 (2002)

[22] X. Ruan et al., Phys. Rev. C 75, 057001 (2007)

[23] H. Witała, private communications, 2018

[24] A. Deltuva, private communications, 2018 